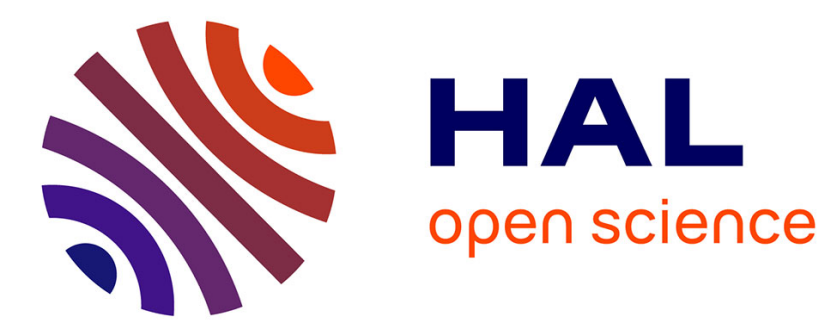

\title{
Le fantasme antidémocratique de l'art total, de Wagner à Mallarmé
}

Frédérik Detue

\section{To cite this version:}

Frédérik Detue. Le fantasme antidémocratique de l'art total, de Wagner à Mallarmé. Revue de Litterature Comparee, 2014, 349 (1), pp.25-38. 10.3917/rlc.349.0025 . hal-02613974

\section{HAL Id: hal-02613974 https://hal.science/hal-02613974}

Submitted on 8 Jun 2020

HAL is a multi-disciplinary open access archive for the deposit and dissemination of scientific research documents, whether they are published or not. The documents may come from teaching and research institutions in France or abroad, or from public or private research centers.
L'archive ouverte pluridisciplinaire HAL, est destinée au dépôt et à la diffusion de documents scientifiques de niveau recherche, publiés ou non, émanant des établissements d'enseignement et de recherche français ou étrangers, des laboratoires publics ou privés. 


\section{Le fantasme antidémocratique de l'art total, de Wagner à Mallarmé}

Le concept de kitsch a été forgé au $\mathrm{XX}^{\mathrm{e}}$ siècle pour critiquer le concept d'art légué par le XIX ${ }^{\mathrm{e}}$ siècle. Dans sa conférence fameuse de 1951 sur le kitsch, Hermann Broch impute d'abord au Grand art romantique la responsabilité du kitsch apparu dans son sillage, puis il analyse que la réaction artistique contre le kitsch de la seconde moitié du XIX ${ }^{\mathrm{e}}$ siècle - celle de l'art pour l'art et de l'œuvre d'art totale - n'a été en fait qu'une pseudo-opposition, voire le phénomène le plus caractéristique de la bifurcation kitsch ${ }^{1}$. Éloigné du marxisme critique, il rejoint pourtant par son analyse les réflexions de Benjamin et d'Adorno sur la collusion qu'il y a au XIX ${ }^{\mathrm{e}}$ siècle entre la «valeur d'art » cultuelle et le fétichisme de la marchandise, spécialement quand «l'idée de l'œuvre d'art totale [...] tente de protéger l'art en le rendant imperméable au développement de la technique $»^{2}$. Dans le sens de Broch qui « consid[ère] Wagner comme [le] plus haut sommet [du kitsch] jamais atteint $»^{3}$, Adorno souligne ainsi que, dans le moment où «le compositeur espère échapper aux exigences mercantiles standardisées de la marchandise “opéra" », sa musique «s'enfonce encore plus profondément dans la marchandise $»^{4}$.

Le problème qui anime ces auteurs est politique, cependant ; s'ils s'intéressent au «destin de l'art au XIX ${ }^{\mathrm{e}}$ siècle », c'est que pour eux «a sonné l'heure fatale de l'art $»^{5}$ : ils ont en tête l'art « dans un sens fasciste » de leur temps, ou ont observé que Hitler, qui « vivait le kitsch sanglant et aimait le kitsch des pièces montées », «a été un partisan absolu du kitsch $»^{6}$. Ce qu'ils dénoncent alors dans l'art pour l'art, c'est surtout un «esprit péremptoire [qui] intensifie l'indifférence sociale jusqu'à la cruauté

\footnotetext{
${ }^{1}$ Voir Hermann Broch, Quelques remarques à propos du kitsch, trad. de l'allemand par Albert Kohn, Paris, Éditions Allia, 2001. Texte d'abord édité en français sous le titre «Quelques remarques à propos de l'art tape-à-l'œil : conférence », dans H. Broch, Création littéraire et connaissance : Essais, trad. de l'allemand par Albert Kohn, Paris, Gallimard, coll. « Tel », 2007, p. 309-325.

${ }^{2}$ Walter Benjamin, «Paris, capitale du XIX siècle: Exposé de 1935 », dans Paris, capitale du XIX ${ }^{e}$ siècle : Le Livre des Passages, trad. de l'allemand par Jean Lacoste, Paris, Les Éditions du Cerf, coll. «Passages », 2000, p. 41.

${ }^{3} \mathrm{H}$. Broch, Quelques remarques à propos du kitsch, op. cit., p. 15.

${ }^{4}$ Theodor W. Adorno, Essai sur Wagner, trad. de l'allemand par Hans Hildenbrand et Alex Lindenberg, Paris : Gallimard, coll. «Les essais », 1966, p. 110.

${ }^{5}$ C'est ce qu'écrivait Walter Benjamin à Max Horkheimer dans une lettre de 1935 : voir la notice de «L'œuvre d'art à l'époque de sa reproduction mécanisée » (1936), dans W. Benjamin, Écrits français, Paris, Gallimard, coll. «Folio/Essais », 2003, p. 158.

${ }^{6} \mathrm{H}$. Broch, Quelques remarques à propos du kitsch, op. cit., p. 35 .
} 
authentique $»^{7}$, car il mène au «mot d'ordre du fascisme », soit, selon Benjamin, «Fiat ars, pereat mundus $»^{8}$.

C'est sous cet angle politique que la théorie et la pratique artistiques de Wagner sont une cible privilégiée. Rendre l'art imperméable au développement de la technique, c'est en général dénier le déclin de l'aura en accusant le « règne de la mode » et «l'égoïsme absolu » de tous les maux de l'humanitée, et c'est vouloir que l'art s'enveloppe du vêtement de la religion de la façon la plus obscurantiste : «quitt[er], une fois pour toutes, le terrain de l'histoire et [s'établir] sur celui de la légende », faire que «la toute-puissante efficacité de la musique» «désarme pleinement la raison logique » au profit $\mathrm{du}$ «sentiment purement humain » $\mathrm{du}$ «Peuple », appeler enfin les hommes à former un grand corps collectif homogène en renouant avec un état humain en harmonie avec « la nécessité du naturel » ${ }^{10}$. Tel est le programme fondamentalement antidémocratique du Gesamtkunstwerk wagnérien, or on connaît son immense succès, dans une époque malade de l'idée de décadence. D'une façon générale, que l'on s'empare de ce Gesamtkunstwerk pour le continuer, ou que l'on relève le «défi » qu'il constitue par une œuvre d'art totale alternative voire anti-wagnérienne, ou enfin que l'on exprime sa nostalgie d'une œuvre d'art totale encore à (re)trouver, - ce sont fausses synthèses en série constamment élaborées en Europe sur fond de wagnérisme - d'une œuvre wagnérienne «prise comme point de référence incontournable, voire indépassable $»^{11}-$, des années 1880 aux années 1920-1930.

\footnotetext{
${ }^{7}$ H. Broch, Création littéraire et connaissance..., op. cit., p. 59.

${ }^{8} \mathrm{~W}$. Benjamin, «L'œuvre d'art à l'époque de sa reproductibilité technique (dernière version de 1939) », dans Euvres, t. III, trad. de l'allemand par M. de Gandillac, R. Rochlitz et P. Rusch, Paris, Gallimard, coll. «Folio/Essais », 2000, p. 316. On peut traduire : «Qu'advienne l'art, le monde dût-il périr ».

${ }^{9}$ Sur la sortie de l'âge d'or grec entraînant « depuis ce moment jusqu'à nos jours » une « histoire de l'égoïsme absolu» et sur le désir d'abolir «les conditions du règne de la mode » pour faire advenir «les conditions de l'art véritable », voir Richard Wagner, L'Euvre d'art de l'avenir [1849], dans Euvres en prose, t. III, trad. de l'allemand par J.-G. Prod'homme et F. Holl, Plan de la Tour, Éditions d'Aujourd'hui, coll. « Les introuvables », 1976, p. 77-78, 192-193.

${ }^{10}$ Sur le parti pris de la légende et l'invocation de la puissance de la musique, voir R. Wagner, "Musique de l'avenir»: Lettre sur la musique [1860], dans ibid., t. VI, p. 210, 219, 225 ; sur la conception wagnérienne du «communisme » comme union de tous ceux qui reconnaissent pour leur l'essence de la nature et qui en conséquence « ressentent une nécessité commune », voir L'Euvre d'art de l'avenir, dans ibid., t. III, p. 60, 68, 84.

${ }^{11}$ Timothée Picard, L'Art total : Grandeur et misère d'une utopie (autour de Wagner), Rennes, Presses universitaires de Rennes, coll. « Esthetica», 2006, p. 12. Point de référence incontournable, voire indépassable, poursuit l'auteur, "sur le triple plan de la totalité théorique des arts, de la totalité concrète de l'œuvre d'art dont la conjonction formelle Tétralogie-Bayreuth reste la manifestation, et de la totalité métaphysique, amenée à exprimer l'essence du monde sans médiation » (ibid.).
} 
Dans ce cadre, il me paraît nécessaire de réévaluer la position de Stéphane Mallarmé, à qui l'on doit « la formulation la plus éclatante du défi wagnérien ${ }^{12}$. Je pense en effet que l'on a surestimé la critique de Wagner par le poète français, et qu'en négligeant en conséquence de considérer ce que son œuvre doit exactement au Gesamtkunstwerk antidémocratique, on s'est peut-être privé des conditions qui permettent de penser avec justesse son articulation à la politique.

On sait l'importance de l'injonction de Mallarmé aux poètes de «rechercher [...] un art d'achever la transposition, au Livre, de la symphonie ou uniment de reprendre [leur] bien ${ }^{13}$. Selon Paul Valéry, ce mot d'ordre, devenu «l'intention commune à plusieurs familles de poètes (d'ailleurs ennemies entre elles) », résume à lui seul « [c]e qui fut baptisé : le Symbolisme », car il est le «principe » d'une poésie qui «se v[eut] réduire à son essence propre »"14, dans sa prétention au «Mystère » (qu'à tort « on veut à la Musique, limiter ${ }^{15}$ ). Or, s'il s'agit, «dans cet acte de juste restitution, qui doit être [celui des poètes], de tout reprendre à la musique », de ne pas «[laisser] s'évanouir le vieux dogme du vers » et, dans son «effort d'orchestration écrite », de ne pas «phras[er] en compositeur, plutôt qu'en écrivain ${ }^{16}$ (comme le fait trop René Ghil au gré de Mallarmé), il apparaît en fait que l'essentialisation symboliste de la poésie ne s'oppose pas au projet de l'œuvre d'art totale, qu'elle est dialectiquement - comme l'envers ou le mode mineur du projet wagnérien (d'un Wagner poète et philosophe «dont on f[ait] l'évangéliste d'un art spirituel, et du symbolisme avant l'heure $»^{17}$ ) mais qu'elle est bien de l'art total. « [C]ela se passe, en toute pureté, sans l'entremise de cordes à boyaux et de pistons comme à l'orchestre, qui est déjà industriel; mais c'est la même chose que l'orchestre, sauf que littérairement ou silencieusement ${ }^{18}{ }^{18}$ expose Mallarmé, et en effet demeure l'idée

\footnotetext{
${ }^{12}$ Ibid., p. 64.

${ }^{13}$ Stéphane Mallarmé, Divagations [1897]: «Crise de vers», dans Euvres complètes, t. II, Paris, Gallimard, coll. «Bibliothèque de la Pléiade », 2003, p. 212.

${ }^{14}$ Paul Valéry, Variété : «Avant-propos à La Connaissance de la Déesse » [1920], dans CEuvres, t. I, Paris, Gallimard, coll. «Bibliothèque de la Pléiade », 1957, p. 1272-1273.

${ }^{15}$ S. Mallarmé, Divagations : «Le Mystère dans les Lettres », dans CEuvres complètes, op. cit., t. II, p. 232 .

${ }^{16}$ S. Mallarmé, «À René Ghil » [7 mars 1885], dans Correspondance complète, 1862-1871; suivi de Lettres sur la poésie, 1872-1898 : Avec des lettres inédites, Paris, Gallimard, coll. « Folio Classique », 1995, p. 577.

${ }^{17}$ Bertrand Marchal, La Religion de Mallarmé : Poésie, mythologie et religion, Paris, J. Corti, 1988, p. 168.

${ }^{18}$ S. Mallarmé, «À Edmund Gosse » [10 janvier 1893], dans Correspondance complète..., op. cit., p. 614. Je souligne.
} 
wagnéro-schopenhaurienne que «l'œuvre la plus complète du poète doit être celle qui, dans son dernier achèvement, serait une parfaite musique ${ }^{19}$, dès lors que 1 'on entend bien «Musique dans le sens grec, au fond signifiant Idée ou rythme entre des rapports ; là, plus divine que dans son expression publique ou symphonique $»^{20}:$ dans ce sens, il est possible d'affirmer que « [1]a Poésie, proche l'idée, est Musique, par excellence $»^{21}-$ qu'elle est, comme la Sainte du vitrail, «Musicienne du silence $»^{22}$.

La Musique que pratique Mallarmé, et qu'il définit encore comme « l'au-delà magiquement produit par certaines dispositions de la parole, où celle-ci ne reste qu'à l'état de moyen de communication matérielle avec le lecteur comme les touches du piano $»^{23}$, c'est l'art poétique de capter le mystère de l'être, ce «quelque chose d'occulte au fond de tous »-ce « quelque chose d'abscons, signifiant fermé et caché, qui habite le commun », auquel il «croi $[\mathrm{t}] »^{24}$. Dans ce sens, son œuvre poétique

\footnotetext{
${ }^{19}$ R. Wagner, "Musique de l'avenir » : Lettre sur la musique, dans CEuvres en prose, op. cit., t. VI, p. 202. Ce propos wagnéro-schopenhaurien devient un topos au tournant du $\mathrm{XX}^{\mathrm{e}}$ siècle. Selon le critique littéraire et historien de l'art anglais Walter Pater, exemplairement, "[t]out art aspire constamment à la condition de la musique » ["All art constantly aspires towards the condition of music"] et «c'est dans la musique [...], plus que dans la poésie, que doit être trouvé le véritable idéal, ou critère, de l'art achevé » ["In music [...], rather than in poetry, is to be found the true type or measure of perfected art"]. (Walter Pater, «The School of Giorgione » [1877], Three Major Texts, New-York, London, New York Univ. Press, 1986, p. 156 et 158. Je traduis.)

${ }^{20} \mathrm{~S}$. Mallarmé, « À Edmund Gosse » [10 janvier 1893], dans Correspondance complète..., op. cit., p. 614.

${ }^{21}$ S. Mallarmé, Divagations : «Quant au livre» («Le Livre, instrument spirituel»), dans Euvres complètes, op. cit., t. II, p. 226.

${ }^{22}$ S. Mallarmé, Poésies : « Sainte », dans ibid., t. I, p. 27.
}

On parle souvent d' «aspirations contradictoires à la totalité et à la pureté », tel Antoine Compagnon qui juge que «le $\mathrm{XX}^{\mathrm{e}}$ siècle, en concurrence avec le rêve romantique de l'œuvre totale, du Gesamtkunstwerk wagnérien, a aussi exigé que chaque art tende vers la pureté de son médium et rejoigne son essence » (Antoine Compagnon, «L'hypertexte proustien », dans Jean Galard, Julian Zugazagoitia (dir.), L'Euvre d'art totale, Paris, Gallimard / Musée du Louvre, coll. " Art et artistes », 2003, p. 93-94). Or il importe de souligner que, si «leurs implications esthétiques diffèrent » en effet, ces tendances relèvent bien néanmoins toutes deux «d'une expression totalisante »; comme l'indique justement Marcella Lista, «[d]ès le XIX ${ }^{\mathrm{e}}$ siècle, dans la dynamique de son développement, l'idée de l'œuvre d'art totale connaît en fait un phénomène de va-et-vient entre l'utopie d'un langage unique et absolu, susceptible de tout contenir en lui-même, et l'aspiration à une synthèse concrète des arts, amenés à se rencontrer dans une forme monumentale » (M. Lista, L'Euvre d'art totale à la naissance des avant-gardes : 1908-1914, Paris, CTHS/INHA, coll. «L'art et l'essai », 2006, p. 9). Voir également J. Zugazagoitia, «Archéologie d'une notion, persistance d'une passion », dans J. Galard, J. Zugazagoitia (dir.), L'Euvre d'art totale, op. cit., p. 72 : «C'est à la fois dans la continuité de cette aspiration à l'œuvre d'art totale et à l'extrême opposé de sa réalisation wagnérienne que se situent certaines expériences d'un art individuel, poussé jusqu'en son essence afin d'arriver au cœur de l'art, là où toutes les formes artistiques se rejoignent et dépassent ce qui semblait d'abord être leur limitation et leur spécificité, à savoir leur matérialité. [...] Mallarmé, Rodin et Monet, tout en étant très éloignés des conceptions d'un Wagner, font en quelque sorte écho aux thèses de celui-ci, par l'ambition fondamentale d'absolu qui les anime ».

${ }^{23}$ S. Mallarmé, « À Edmund Gosse » [10 janvier 1893], dans Correspondance complète..., op. cit., p. 614.

${ }^{24}$ S. Mallarmé, Divagations : «Le Mystère dans les Lettres », dans Euvres complètes, op. cit., t. II, p. 229-230. 
participe d'une tendance qui entreprend de réélaborer le projet wagnérien, non pas suivant le modèle athénien de la tragédie sur lequel se fonde explicitement ce projet, mais suivant le modèle, médiéval, du mystère chrétien - qu'il lui substitue moyennant une conversion de la Passion du Christ (du Dieu fait homme) en celle de l'Homme (de l'homme-dieu). Cependant, ce n'est pas seulement l'art du poète lyrique, chez les symbolistes, que d'éveiller l'homme intégral des origines, spécialement grâce aux associations synesthésiques. La proposition de transformer le projet wagnérien en art du roman, conçu comme idéal esthétique et métaphysique, détermine un enjeu analogue, quand, sous le signe de Wagner, on en vient à envisager le roman de l'intériorité dans la Revue wagnérienne ${ }^{25}$. Prônant en général «non pas tant l'œuvre d'art totale, que la totalité au sein de chaque art, en tant que cette totalité est [...] celle de l'homme complet », Teodor de Wyzewa appelle en effet de ses vœux « un roman de vie complète, tout ensemble descriptif et psychologique ${ }^{26}$, or, suivant Édouard Dujardin, cette littérature «fondamentalement Wagnérienne» capable de «rendre compte artistiquement de l'intégralité de ce qui fait l'homme» doit être la « [q]uintessence de la vie même » et par conséquent «[capter] le mystère de l'être », en tant que littérature «absolument suggestive» qui «aura de très mystérieux palpitements d'universelle Clairvoyance $»^{27}$. Quant au théâtre, Maurice Maeterlinck juge que «la bonne musique » - entendons : wagnérienne - lui ouvre également tout un horizon de vie « en profondeur, en signification intime et en gravité spirituelle » en ce qu'elle sait «démêler et reproduire les traits les plus cachés, mais non moins graves et étonnants de la vie d'aujourd'hui $»^{28}$. Selon lui, le drame moderne doit ainsi exprimer à son tour « quelque chose de la vie rattachée à ses sources et à ses mystères par des liens [qu'on n'a] l'occasion ni la force d'apercevoir tous les jours » ${ }^{29}$, ce en

\footnotetext{
${ }^{25}$ D'un point de vue aussi bien sociologique que théorique, la filiation de Wagner au symbolisme français a été assurée notamment par la création de cette Revue wagnérienne (1885-1888); comme le note Bertrand Marchal à propos du fondateur de la revue Édouard Dujardin, celui-ci, «si l'on en croit ses souvenirs, avait conscience que la revue était, autant qu'un instrument de promotion du wagnérisme, un manifeste littéraire à peine déguisé, comme la préface de Cromwell d'un symbolisme à naitre, puisqu'il avait voulu lui offrir un parrain dont le moins qu'on puisse dire est que ses compétences en matière de wagnérisme ne l'imposaient pas à cette place : Mallarmé lui-même » (B. Marchal, La Religion de Mallarmé..., op. cit., p. 169).

${ }^{26}$ T. Picard, L'Art total..., op. cit., p. 101-102. Voir Teodor de Wyzewa, « Notes sur la littérature wagnérienne et les livres en 1885-1886», dans Revue wagnérienne ( $\mathrm{n}^{\circ} \mathrm{V}, 8$ juin 1886), t. II : 18861887, Genève, Slatkine reprints, 1993, p. 150-171.

${ }^{27}$ T. Picard, L'Art total..., op. cit., p. 104. Voir Édouard Dujardin, «Chronique (Akëdysséril)», dans Revue wagnérienne ( $\mathrm{n}^{\circ}$ VII, 8 août 1885), op. cit., t. I : 1885-1886, p. 193-194.

${ }_{28}$ Maurice Maeterlinck, «Le tragique quotidien» [1896], CEuvres, t. I, Bruxelles, Complexe, coll. «Bibliothèque Complexe », 1999, p. 488.

${ }^{29}$ M. Maeterlinck, «Le tragique quotidien » [1896], CEuvres, op. cit., t. I, p. 489.
} 
quoi la tâche du poète dramatique se révèle plus ardue que celle du poète lyrique, à qui «[i]l est peut-être loisible [...] de demeurer une sorte de théoricien de l'inconnu » : car, loin de se borner à des généralités, le poète dramatique « est obligé de faire descendre dans la vie réelle, dans la vie de tous les jours, l'idée qu'il se fait de l'inconnu. Il faut qu'il nous montre de quelle façon, sous quelle forme, dans quelles conditions, d'après quelles lois, à quelle fin, agissent sur nos destinées, les puissances supérieures, les influences inintelligibles, les principes infinis, dont, en tant que poète, il est persuadé que l'univers est plein $»^{30}$.

Le credo métaphysique ici exposé n'est pas éloigné de celui des premiers romantiques allemands, pour qui tout réel est un être pensant, et c'est très logiquement que, dans 1' «Introduction» à sa traduction des Disciples à Saïs de Novalis, Maeterlinck détermine cette tâche du poète comme l'action de romantiser. Évoquant «notre conscience à peu près inconsciente», il affirme en effet qu'«[a]ugmenter cette conscience transcendantale semble avoir été toujours le désir inconnu et suprême des hommes $»^{31}$ : c'est ainsi que «[le poète] est plus ou moins puissant, non pas en raison de ce qu'il fait lui-même, mais en raison de ce qu'il parvient à faire exécuter par les autres, et par l'ordre mystérieux et éternel et la force occulte des choses ${ }^{32}$. À partir des années 1880 , cependant, le critérium de la puissance artistique étant l'organon wagnérien, cette sacralisation de la littérature et de l'art suivant le modèle médiéval du mystère nourrit un rêve - tenace - de cathédrale. $\mathrm{Si}$ « la cathédrale est un lieu très prisé » en cette fin de siècle ${ }^{33}$, ce n'est pas tant la référence à l'édifice réel qui prime, que celle au symbole d'unité et de transcendance qu'il représente. Éditée en 1898, la thèse d'Émile Mâle sur L'Art religieux du XIII siècle en France est symptomatique de cette tendance : l'auteur se concentre en effet sur « la révélation totale ${ }^{34}$ qu'était la cathédrale pour les hommes du Moyen Âge, en tant que «pensée faite pierre et verre « «ont pas un élément

\footnotetext{
${ }^{30}$ M. Maeterlinck, « Préface au Théâtre de $1901 »[1901]$, dans ibid., p. 500.

${ }^{31}$ M. Maeterlinck, «"Introduction” à la traduction des Disciples à Saïs de Novalis » [1895], dans ibid., p. 291.

${ }^{32}$ M. Maeterlinck, « Réponse à l'Enquête de Jules Huret » [1891], dans ibid., p. 587.

${ }^{33}$ Comme le montre, exemples à l'appui, Jean de Palacio : voir «La cathédrale décadente : monstre hybride et repaire de monstres », dans Joëlle Prungnaud (dir.), La Cathédrale, Villeneuve-d'Ascq, Université Charles-de-Gaulle - Lille 3, CeGes, coll. «UL3 Travaux et recherches », 2001, p. 137-138.

${ }^{34}$ Émile Mâle, L'Art religieux au XIII ${ }^{e}$ siècle en France, Paris, Librairie générale française, coll. « Le livre de poche, Biblio Essais », 1987, p. 713.
} 
n'échappe à l'intention unitaire de l'Église ${ }^{35}$. Ainsi, la cathédrale comme Somme pour la scolastique du Moyen Âge tend à se métamorphoser en livre, en cette époque en quête de sacré hors des sentiers religieux institués.

Tandis que Wagner concevait le Gesamtkunstwerk comme un moyen de «réparer Babel ${ }^{36}$ au Palais des Festivals de Bayreuth, Dujardin va jusqu'à considérer l'accomplissement wagnérien de l'idéal babélien comme Livre : dans le troisième d'une série d'articles sur Bayreuth, il juge que la forme théâtrale n'est en fait «qu'un compromis » fait pour « rendre lisible au plus grand nombre cette bible nouvelle » qu'est la partition d'orchestre, et il « tir[e], dans une étrange confusion des théories wagnériennes et des idées de Mallarmé, le concept de l'œuvre vers celui de Livre, ou de Bible d'une nouvelle religion, mais un livre accessible à quelques rares initiés, capables de lire à même la partition ${ }^{37}$. Cependant, cette proposition n'est pas du goût de Mallarmé, qui, faisant grief à la partition du compositeur de mal taire sa musique d'orchestre, récuse l'idée d'un Livre wagnérien ${ }^{38}$. Selon le grand prêtre des Mardis de la rue de Rome, en effet, il faut continuer de penser que le défi lancé par Wagner gît dans la formule, musicale et théâtrale, du drame et dans celle-ci seulement, et que la formule du Livre constitue une riposte poétique. C'est en cela que, suivant le commentaire de Wyzewa, Mallarmé « renonce l'Art, et s'occupe à la Religion, méditant, comme déjà Wagner, une œuvre d'universelle Révélation métaphysique ${ }^{39}$. Car, l'objectif de sa riposte poétique étant de «concurrencer, dans un avenir indéfini, les pompes annuelles de Bayreuth $»^{40}$, il se prend à rêver, comme

\footnotetext{
${ }^{35}$ Luc Fraisse, «D'Émile Mâle à Proust: Comment la cathédrale devient le symbole de la Recherche », dans J. Prungnaud (dir.), La Cathédrale, op. cit., p. 189 et 186. Voir É. Mâle, L'Art religieux au XIII siècle en France, op. cit., p. 703 : l'auteur cite Victor Hugo affirmant dans NotreDame de Paris qu' « [a]u Moyen Âge, le genre humain n'a rien pensé d'important qu'il ne l'ait écrit en pierre », puis il commente : «Victor Hugo a dit vrai : la cathédrale est un livre. C'est à Chartres que ce caractère encyclopédique de l'art du Moyen Âge est le mieux marqué [...]. La cathédrale de Chartres est la pensée même du Moyen Âge devenue visible ».

36 J. Zugazagoitia, «Archéologie d'une notion, persistance d'une passion », dans J. Galard, J. Zugazagoitia (dir.), L'Euvre d'art totale, op. cit., p. 70. Dans L'Euvre d'art de l'avenir, Wagner compare la chute de l'humanité que représente la fin de l'âge d'or grec à celle que représente l'épisode de la tour de Babel : voir R. Wagner, L'Euvre d'art de l'avenir, dans CEuvres en prose, op. cit., t. III, p. 110.

37 B. Marchal, La Religion de Mallarmé..., op. cit., p. 196. Voir É. Dujardin, «Bayreuth (Fin) : Théories wagnériennes », dans Revue wagnérienne ( $\mathrm{n}^{\circ}$ VII, 8 août 1885), op. cit., t. I : 1885-1886, p. 206-210.

${ }^{38}$ Dans son «Hommage » : «Le dieu Richard Wagner irradiant un sacre / Mal tu par l'encre même en sanglots sibyllins. » (S. Mallarmé, Poésies : «Hommage »[Revue wagnérienne, $\mathrm{n}^{\circ} \mathrm{XII}, 8$ janvier 1886], dans Euvres complètes, op. cit., t. I, p. 40.)

${ }^{39}$ T. de Wyzewa, «La religion de Richard Wagner et la religion du comte Léon Tolstoï », dans Revue wagnérienne ( $\mathrm{n}^{\circ}$ VIII-IX, 8 octobre 1885), op. cit., t. I : 1885-1886, p. 238, note 3.

${ }^{40}$ B. Marchal, La Religion de Mallarmé..., op. cit., p. 519.
} 
«Grand Euvre » (alchimique), « un livre, architectural et prémédité, et non un recueil des inspirations de hazard [sic], fussent-elles merveilleuses $»^{41}-$ un livre-cathédrale, autrement dit, et non un livre-«cloître [...] brisé » tel que Divagations en 1897, ce « livre comme [il] ne les aime pas, ceux épars et privés d'architecture » ${ }^{42}$.

Divagations est un livre propre à un temps d'«interrègne », de coupure entre poésie et société, de solitude du poète d'une part, et d'anomie sociale d'autre part ; il procède d'une morale provisoire de l'« action restreinte ». Mais il faut se projeter audelà de cet interrègne, dans la croyance «que la poésie est faite pour le faste et les pompes suprêmes d'une société constituée où aurait sa place la gloire dont les gens semblent avoir perdu la notion ${ }^{43}$. Cette autre époque ontologique est « un jour qui gît au sein, inconscient, de la foule ${ }^{44}$, un «Présent » qui «n'existe pas[,] [f]aute que se déclare la Foule $»^{45}$, - mais donc un temps qui pourrait advenir enfin, quand ce qui n'est, suivant la malédiction babélienne, que multitude dispersée sur la surface de la terre aurait perdu de son inconsistance pour former une «Foule». On observe ici le chemin parcouru par Mallarmé depuis son exhortation au poète de « rester aristocrate » et de ne pas livrer sa poésie au «Philistin », en $1862^{46}$. Déjà en 1877 , « [i]l fallait bien toute la force de suggestion de l'idéal wagnérien et l'exemple de la volonté d'un homme qui avait réussi à imposer cet idéal à ses contemporains, pour que le poète élitiste qui dénonçait l'art pour tous comme une hérésie artistique [...], rêvât quinze ans plus tard d'un théâtre "qui éblouisse le peuple souverain comme ne le fut jamais empereur de Rome ou prince d'Asie", et se découvrît ainsi sur le tard une âme d'artiste populaire ${ }^{47}$. Or cette force de suggestion se fait sentir plus nettement

\footnotetext{
${ }^{41}$ S. Mallarmé, «À Paul Verlaine » [16 novembre 1885], dans Correspondance complète..., op. cit., p. 585.

${ }_{42}^{2}$ S. Mallarmé, Divagations, dans Euvres complètes, op. cit., t. II, p. 82. «"CC]loître brisé", ditesvous, non : cathédrale sur le papier, aux beautés morcelées, parce qu'elle ne peut s'y étaler toute », écrit Georges Rodenbach à Mallarmé le 28 janvier 1897 ; mais c'est qu'il a en vue « une cathédrale décadente », soit un «livre, comme il se doit, brisé, privé et morcelé » dont «chaque poète futur $[\ldots]$ volera un morceau pour le porter avec lui - relique, modèle de beauté, pierre sculptée où se résume une tour que chacun continue... » (voir J. de Palacio, «La cathédrale décadente : Monstre hybride et repaire de monstres », dans J. Prungnaud (dir.), La Cathédrale, op. cit. p. 143 ; voir également L'Amitié de Stéphane Mallarmé et de Georges Rodenbach, Genève, Pierre Cailler Éditeur, 1949, p. 98).

${ }^{43}$ S. Mallarmé, Entretiens : «Sur l'évolution littéraire [Enquête de J. Huret] », dans Euvres complètes, op. cit., t. II, p. 700.

${ }^{44}$ S. Mallarmé, Divagations : «Richard Wagner : Rêverie d'un Poëte français » [Revue wagnérienne, $\mathrm{n}^{\circ}$ VII, 8 août 1885], dans ibid., p. 153.

${ }^{45}$ S. Mallarmé, Divagations : «Quant au livre » («L'action restreinte »), dans ibid., p. 217.

${ }^{46}$ Voir S. Mallarmé, Articles : «Hérésies artistiques : L’art pour tous », dans ibid., p. 360-364.

${ }^{47}$ B. Marchal, La Religion de Mallarmé..., op. cit., p. 172. Voir S. Mallarmé, Correspondance complète..., op. cit., p. 564. B. Marchal cite une lettre de Mallarmé à Arthur O'Shaughnessy du 28 décembre 1877 en note : «je travaille follement; et j'étudie partout les fragments d'un Théâtre
} 
encore relativement à l'utopie religieuse du Livre, à laquelle «[1]a Foule, avec une majuscule significative, est [...] ce que le peuple, Volk, est au drame wagnérien, à la fois le moyen et la fin de l'art authentique ${ }^{48}$. D'un côté - par la Foule comme moyen -, « en la foule ou amplification majestueuse de chacun gît abscons le rêve ${ }^{49}$, c'est-à-dire que le prestige de la foule mallarméenne tient à «sa fonction par excellence de gardienne du mystère $»^{50},-$ fonction de médiation religieuse à travers laquelle tout homme peut retrouver le sens de sa grandeur; ainsi, c'est «dans un temps véritablement advenu comme l'ère de la foule » qu' « il y a [...], pour le poète, une chance même improbable pour que "ce doive illuminer", pour que des vérités symboliques sur l'homme et sur le monde puissent enfin "éclater, diamantairement [...], en l'intégrité du Livre" » ${ }^{51}$. De l'autre - pour la Foule comme fin -, celle-ci «n'existe qu'en relation avec un art qui la constitue, un art du moins qui se lie luimême en quelque façon au mystère originel de l'homme et du ciel » ${ }^{52}$, c'est-à-dire que la fonction de médiation religieuse se déplace : « la foule, quand elle aura, en tous les sens de la fureur, exaspéré sa médiocrité [...], hurlera vers le poëte, un appel », de sorte que c'est du poète, «détenteur de la splendeur commune, auquel on sera bien forcé de recourir », que doit venir, grâce à «son feu d'artifice [tiré] sur la place publique », «l'explication, même humaine, de ce rassemblement » ${ }^{53}$. Mallarmé imagine donc dans ce sens une « récitation » poétique publique qui soit «comme un aboutissement à l'ère moderne, esthétique et industrielle, de tout le jet forcément par la Renaissance limité à la trouvaille technique », et qui soit en conséquence «comme l'Ouverture d'un Jubilé, notamment de celui au sens figuratif qui, pour conclure un cycle de l'Histoire, [lui] semble exiger le ministère du Poëte »- une « récitation » qui « charmera, instruira [...] et par-dessus tout émerveillera le Peuple ${ }^{54}$ :

Dans une fin de siècle où les prêtres en tout genre perdent leur autorité symbolique sur la foule, tandis que les ouvriers n'ont de choix qu'entre l'usine cette autre tombe - et le nihilisme des bombes anarchistes, le poète fait ainsi de

nouveau qui se prépare en France et que je prépare de mon côté ; quelque chose qui éblouisse le peuple souverain comme ne le fut jamais empereur de Rome ou prince d'Asie ».

${ }^{48}$ B. Marchal, La Religion de Mallarmé..., op. cit., p. 526.

49 S. Mallarmé, Divagations : «Crayonné au théâtre ( «Crayonné au théâtre »), dans Euvres complètes, op. cit., t. II, p. 165.

${ }^{50}$ S. Mallarmé, Divagations : « Offices » (« Plaisir sacré »), dans ibid., p. 237.

${ }^{51}$ B. Marchal, La Religion de Mallarmé..., op. cit., p. 529. Voir S. Mallarmé, Divagations : « Quant au livre » («L'action restreinte »), dans Euvres complètes, op. cit., t. II, p. 218.

${ }^{52}$ B. Marchal, La Religion de Mallarmé..., op. cit., p. 527.

${ }^{53}$ S. Mallarmé, Villiers de l'Isle-Adam [1892], III, dans CEuvres complètes, op. cit., t. II, p. 40.

${ }^{54}$ S. Mallarmé, Divagations : «Crayonné au théâtre » («Solennité »), dans ibid., p. 203. 
la liturgie publique du Livre le lieu d'émergence d'un nouveau type de héros moderne, prêtre et ouvrier, qui justifie un sacerdoce nouveau - le ministère du poète - par une bombe toute symbolique, le feu d'artifice de l'œuvre. ${ }^{55}$

Quant à ce rêve de «communion, par le livre » ${ }^{56}$, Mallarmé l'a fondamentalement conçu suivant le modèle d'une liturgie publique inspirée par les fastes des célébrations nationales (et même si, eu égard aux conditions de l'interrègne, il a pu dans les années 1890 substituer à ce premier modèle celui, de tradition aristocratique, de la séance académique ou du rituel des sociétés savantes). C'est ce dont témoigne sa première idée d'un «ministère du poëte, en 1889 » $^{57}$, comme si la Révolution française devait «s'achever, un siècle plus tard, dans une révolution spirituelle grâce à laquelle la multitude émancipée par 1789, enfin constituée en foule et consciente d'elle-même, ouvrirait une ère nouvelle de l'humanité $»^{58}$. Or loin s'en faut que l'on puisse qualifier cette révolution spirituelle de «démocratique », comme le suggère Bertrand Marchal lorsqu'il soutient que le public visé par «cette apocalypse historique en forme de récitation » est «le peuple entier, moins le Volk que la collectivité civique qui a fait la Révolution » ou que l'enjeu n'est autre que le passage de « la démocratie politique, dont Mallarmé ne conteste pas la légitimité » à « une démocratie religieuse encore à fonder $»^{59}$.

En réalité, si l'on ne peut certes pas trouver chez Mallarmé «l'ébauche d'un projet politique précis », étant donné son souci de ne pas «tomber dans le travers qu'il dénonce, celui d'entretenir la vaine inflation du discours politicien ${ }^{60}$, on peut néanmoins déterminer sans hésitation que tout son rêve de religion du livre, ou de religion par le livre, est fondamentalement dirigé contre la démocratie républicaine, ce dont témoigne d'ailleurs son aversion même pour le discours politique. Ce que le poète appelle «interrègne », en effet, n'est autre que l'ère démocratique, or il en a parfaitement saisi l'essence, quand il évoque la ville de l'interrègne comme de

\footnotetext{
${ }_{55}^{55}$ B. Marchal, La Religion de Mallarmé..., op. cit., p. 511.

${ }^{56} \mathrm{~S}$. Mallarmé, La Musique et les Lettres [1894], dans Euvres complètes, op. cit., t. II, p. 76.

${ }^{57}$ S. Mallarmé, Divagations : Notes et variantes, dans ibid., p. 1642 : B. Marchal expose dans une note que «[1]a version préoriginale » de l'extrait de «Solennité » qui évoque «l'Ouverture d'un Jubilé » devant conclure « un cycle de l'Histoire » "donnait une identité plus précise à ce cycle de l'Histoire. Les derniers mots appelaient en effet "le ministère du Poëte, en 1889", à la date du centenaire de la Révolution ».

${ }_{58}^{58}$ B. Marchal, La Religion de Mallarmé..., op. cit., p. 535.

59 Ibid., p. 535 et 528. Le modèle de la récitation publique est explicitement qualifié de «démocratique » par l'auteur (ibid., p. 537). L'idée de « démocratie religieuse » apparaît cependant comme oxymorique.

${ }^{60}$ Ibid., p. 374.
} 
« [1]ongs faubourgs prolongés par la monotonie de voies jusqu'au central rien » ${ }^{61}$, par opposition à la ville du Moyen Âge dont les voies convergent vers la cathédrale comme vers son centre originaire et fondateur. Une société organique comme le Moyen Âge avait besoin d'un tel monument «extraordinaire, divin ou totalement jailli du sol factice $»^{62}$, qui incarne en son centre la source de la loi, mais qu'est-ce que la démocratie, sinon ce qui précisément mine la représentation d'une société organique en rendant la source de la loi inlocalisable ? Comme le souligne Claude Lefort :

La démocratie libérale naît du rejet de la domination monarchique, de la découverte collectivement partagée que le pouvoir n'appartient à personne, que ceux qui l'exercent ne l'incarnent pas, qu'ils ne sont que dépositaires de l'autorité publique, temporairement, que ne s'investit pas en eux la Loi - celle de Dieu ou celle de la Nature -, qu'ils ne détiennent pas le savoir dernier de l'ordre du monde et de l'ordre social et ne sont pas en mesure de décider de ce que chacun est en droit de faire, de penser, de dire et d'entendre. ${ }^{63}$

Or le « central rien » démocratique représente une faillite sociale, pour Mallarmé :

La Société, terme le plus creux, héritage des philosophes, a ceci, du moins, de propice et d'aisé que rien n'existant, à peu près, dans les faits, pareil à l'injonction qu'éveille à l'âme son concept auguste, en discourir égale ne traiter aucun sujet ou se taire par délassement. Quelque chose, manquant, affronte la violence des contradictions et, dans aucun sens, nous ne risquons de donner trop à fond, sur une entité. Néant ou éclat dans le vide, avec une peur chez la masse accourue au faux abri, tout agencement vulgaire se mettant sous cette invocation profitable. $^{64}$

La société telle que l'a conçue Rousseau, fondée sur un seul contrat social et non sur « une entité », ne peut être qu' «une société sans stabilité, sans unité », «une organisation sociale inachevée $»^{65}$ : sa légitimité est fausse, puisqu'elle s'en remet à l'arbitraire inquiet du vote au suffrage universel - qu'elle « [s']adonn[e] à la foule ou hasard ${ }^{66}$; un pacte social qui «n'exhib[e] point de sceau » est voué à devenir un «pacte déchiré ${ }^{67}$, comme en témoigne «la violence des contradictions ».

\footnotetext{
${ }^{61}$ S. Mallarmé, Divagations : « Grands faits divers » («Bucolique »), dans EEuvres complètes, op. cit., t. II, p. 253.

${ }^{62}$ Ibid.

${ }^{63}$ Claude Lefort, La Complication : Retour sur le communisme. Paris, Fayard, 1999, p. 145-146.

${ }^{64}$ S. Mallarmé, Divagations : «Grands faits divers » («Sauvegarde»), dans CEuvres complètes, op. cit., t. II, p. 271.

${ }^{65}$ S. Mallarmé, Entretiens : «Sur l'évolution littéraire [Enquête de J. Huret] », dans ibid., p. 697-698.

${ }^{66}$ S. Mallarmé, Divagations : «Grands faits divers » (« Bucolique »), dans ibid., p. 252.

${ }^{67}$ S. Mallarmé, Divagations : «Crayonné au théâtre » («Le Genre ou des Modernes »), dans ibid., p. 181.
} 
L'effondrement des ordres autoritaires - monarchique et impérial - a ouvert un temps de liberté démocratique que Mallarmé ne pense ainsi que négativement, comme un temps de «crise » à dépasser dans une nouvelle positivité, de la même façon qu'il pense l'effondrement de l'ordre catholique en religion, ou celui de l'ordre hugolien en poésie ; car, avec la démocratie républicaine comme avec le protestantisme ou la « crise de vers », c'est toujours l' « inexpliqué besoin d'individualité » ${ }^{68}$ qui l'emporte, vouant la vie sociale à l'atomisation et à la vacuité. Contre l'institution du conflit démocratique, il faut donc rétablir de «sociales bases » qui permettent de «[donner] sur une entité », «dou[er] ainsi d'authenticité notre séjour » ${ }^{69}$ en «imprim[ant] à la terre le sceau universel $»^{70}$; et c'est dire que, contre «le fait que nul ne puisse se présenter comme détenteur de la connaissance de l'ordre social et des fins de la conduite humaine $»^{71}$ - et contre les libertés individuelles semeuses de désordre -, il faut réarticuler le théologique et le politique, et subordonner celui-ci à celui-là : ce qu'est censé assurer «le ministère du Poëte », puisque le poète est «détenteur de la splendeur commune ».

À la manière de Wagner, en effet, Mallarmé, «civilisé édennique » ${ }^{72}$, rejette l'inconcilié contemporain comme l'ère de ceux qui ne ressentent aucune nécessité commune, dans l'oubli où ils sont de la nature, et, face à cette amnésie ontologique de nature à favoriser le «besoin d'individualité », fonde tout son projet littéraire et religieux de réparer le «grand dommage [...] causé à l'association terrestre » sur un déni $\mathrm{du}$ «terre-plein »- «niveau élémentaire de la politique ${ }^{73}$-, ne s'intéressant qu'à « ce qui concerne l'homme, seul et dans un raccourci, vis-à-vis du monde » ${ }^{74}$, et visant à promouvoir «les formalités édictant un culte populaire, comme représentatives - de la Loi, sise en toute transparence, nudité et merveille ${ }^{75}$. Il faut partir du principe, selon lui, qu'« $[\mathrm{u}] \mathrm{n}$ gouvernement mirera, pour valoir, celui de l'univers ${ }^{76}$; aussi propose-t-il, pour «s'exiler très haut» du «terre-plein ${ }^{77}$ et

\footnotetext{
${ }^{68}$ S. Mallarmé, Entretiens : «Sur l'évolution littéraire [Enquête de J. Huret] », dans ibid., p. 698.

${ }^{69}$ S. Mallarmé, « À Léo d'Orfer» [27 juin 1884], dans Correspondance complète..., op. cit., p. 572.

${ }^{70}$ S. Mallarmé, Divagations : «Richard Wagner: Rêverie d'un Poëte français », dans Euvres complètes, op. cit., t. II, p. 158.

${ }^{71}$ C. Lefort, La Complication..., op. cit., p. 192.

${ }^{72}$ S. Mallarmé, La Musique et les Lettres, dans Euvres complètes, op. cit., t. II, p. 66.

${ }^{73}$ Ibid., p. 74.

${ }^{74}$ S. Mallarmé, Réponses à des enquêtes : «Sur la meilleure condition du bien social » [1893], dans ibid., p. 660.

${ }^{75}$ S. Mallarmé, La Musique et les Lettres, dans ibid., p. 74.

${ }^{76}$ Ibid., p. 76.

${ }^{77}$ S. Mallarmé, Divagations : « Offices » («Catholicisme »), dans ibid., p. 239.
} 
rendre visible définitivement la correspondance entre l'ordre civique et l'ordre cosmique, de substituer au « central rien » démocratique un «édifice de haut verre », nouveau centre symbolique de la cité : «la gare toute puissante du virginal palais central, qui couronne $»^{78}$. Dans la cathédrale médiévale, écrit Émile Mâle, nous nous trouvons dans « un monde transfiguré où la lumière est plus éclatante que celle de la réalité », où «[1]'homme, enfermé dans une classe sociale, dans un métier, dispersé, émietté par le travail de tous les jours et par la vie, [...] repren[d] le sentiment de l'unité de sa nature », où «[1]a foule, assemblée pour les grandes fêtes, sen[t] qu'elle [est] elle-même l'unité vivante »- et en somme, « [d]éjà nous nous sentons au sein de la Jérusalem céleste, de la cité future ${ }^{79}$. De façon analogue, sous «le vitrage de coupole constatant élévation et transparence $»^{80}$, la cité doit être illuminée par la loi immuable de la nature scellée par l'évidence solaire, la religion ressurgie, permettre «l'amplification à mille joies de l'instinct de ciel en chacun ${ }^{81}$, et les fêtes, faire qu' « un peuple témoigne de sa transfiguration en vérité » ${ }^{82}$. La coupole du « virginal palais central» évoque ici celle de «[1]a plus haute institution» qu'est selon Mallarmé «l'Académie» (française), supérieure à «la Chambre représentative, directe, du pays » ${ }^{83}$, garante d'un « art stable », d'un « art définitif ${ }^{84}$, vu que « son dessein » est de «chang[er] en splendeur officielle l'effort divers » ${ }^{85}$; ainsi, l'« édifice de haut verre » permet d'espérer enfin « la célébration des poèmes étouffés dans l'œuf de quelque future coupole manquant ${ }^{86}$, puisque le rôle du poète de gardien des valeurs et de la puissance de l'esprit y sera étendu à l'échelle de l'État, par un «ministère » qui fera exister la société à hauteur de son «concept auguste», grâce à « un couronnement par l'art » ${ }^{87}$.

Telle est la version mallarméenne du Gesamtkunstwerk antidémocratique. Le poète français a énoncé une critique de celui-ci qui rappelle le propos nietzschéen du Cas Wagner en 1888, lorsqu'il reproche au compositeur l'instrumentalisation de la musique, la violence contre la raison et l'« anachronisme » de la Légende, lorsqu'il

\footnotetext{
${ }^{78}$ S. Mallarmé, Divagations : «Quant au livre » (« L'action restreinte »), dans ibid., p. 217.

${ }^{79}$ É. Mâle, L’Art religieux au XIII ${ }^{e}$ siècle en France, op. cit., p. 713-714.

${ }^{80}$ S. Mallarmé, Divagations : «Offices » («Catholicisme»), dans CEuvres complètes, op. cit., t. II, p. 241.

${ }^{81}$ S. Mallarmé, La Musique et les Lettres, dans ibid., p. 74.

${ }^{82}$ S. Mallarmé, Divagations : «Quant au livre » (« L'action restreinte »), dans ibid., p. 216.

${ }^{83}$ S. Mallarmé, Divagations : «Grands faits divers » (« Sauvegarde »), dans ibid., p. 268.

${ }^{84} \mathrm{~S}$. Mallarmé, Entretiens : «Sur l'évolution littéraire [Enquête de J. Huret] », dans ibid., p. 698.

${ }^{85}$ S. Mallarmé, Divagations : «Grands faits divers » (« Sauvegarde »), dans ibid., p. 268.

${ }^{86}$ S. Mallarmé, Divagations : «Crayonné au théâtre » («Crayonné au théâtre »), dans ibid., p. 165.

${ }^{87}$ S. Mallarmé, Divagations : « Grands faits divers » (« Bucolique »), dans ibid., p. 253.
} 
dénonce non seulement un art de la rêverie mensonger mais encore, un obscurantisme « germain ». Cependant, c'est là essentiellement - comme chez Nietzsche - la critique d'un décadentisme wagnérien, c'est-à-dire qu'il ne s'agit pas, pour Mallarmé, de réfuter l'idéal de l'art wagnérien (comme le fera la critique du kitsch au $\mathrm{XX}^{\mathrm{e}}$ siècle), mais de corriger la réalisation de cet idéal. À cet égard, il est symptomatique qu'il invoque «l'esprit français, strictement imaginatif et abstrait», soumis à une «logique éternelle », dans l'idée qu'un art animé de cet «esprit français » universaliste doit s'opposer à l'« incontestable portique » wagnérien qui «ouvre [...], en des temps de jubilé qui ne le sont pour aucun peuple, une hospitalité contre l'insuffisance de soi et la médiocrité des patries ${ }^{88}$; car, s'il s'en prend ce faisant au second Wagner, suivant lequel l'art vise à concevoir un âge d'or «national et ethnique », il rejoint à son insu la position du premier Wagner, lequel défendait bien avant lui l'idéal d'un âge d'or «non national universel ${ }^{89}$. Ainsi, loin de reprocher au compositeur son projet de produire une belle totalité artistique qui se donne à contempler comme une pure apparence naturelle (comme dans la critique de la fantasmagorie chez Benjamin et Adorno), Mallarmé lui reproche l'impureté légendaire de son Gesamtkunstwerk incapable d'atteindre à cette «visée initiale », et oppose à celui-ci «la Fable, vierge de tout, lieu, temps et personne sus, [...] empruntée au sens latent en le concours de tous, celle inscrite sur la page des Cieux et dont l'Histoire même n'est que l'interprétation, vaine, c'est-à-dire un Poème, l'Ode $»^{90}$.

C'est dans cet esprit que Mallarmé, qui se sait « exclu de toute participation aux déploiements de beauté officiels » et condamné à «de solitaires Fêtes »dans «l'interrègne », « aime [...] à réfléchir aux pompes souveraines de la Poésie, comme elles ne sauraient exister concurremment au flux de banalité charrié par les arts dans le faux semblant de civilisation ${ }^{91}$. Les « [p] avoisements » et «lueurs » en 1889, ce sont ceux de «l'Exposition inoubliable pour beaucoup d'ici » ${ }^{92}$, et en l'occurrence, tandis que les foules participent gaiement aux fêtes du centenaire de la Révolution et

\footnotetext{
${ }^{88}$ S. Mallarmé, Divagations : «Richard Wagner : Rêverie d'un Poëte français », dans ibid., p. 156-158. ${ }^{89}$ R. Wagner, L'Euvre d'art de l'avenir, dans Euvres en prose, op. cit., t. III, p. 88. C'est à partir des années 1860 , et bien plus encore après la guerre franco-prussienne de 1870 et la fondation par Bismarck du II $^{\mathrm{e}}$ Reich allemand en 1871, que Wagner opère un virage de l'universel au national et se met à prôner un destin proprement allemand de la « communauté du peuple » : c'est alors l'époque des Maîtres chanteurs de Nuremberg (1868) et de la réalisation du projet de Bayreuth (achevé en 1874).

${ }^{90}$ S. Mallarmé, Divagations : «Richard Wagner: Rêverie d'un Poëte français », dans Euvres complètes, op. cit., t. II, p. 157.

${ }^{91}$ Ibid., p. 153.

${ }^{92}$ S. Mallarmé, Villiers de l'Isle-Adam, III, dans ibid., p. 39.
} 
se pénètrent de la valeur d'échange des marchandises au point de s'identifier avec elle, le «détenteur de la splendeur commune»par excellence, en la personne de Villiers de l'Isle-Adam, agonise dans l'indifférence générale. Mais - comme «dans l'oubli d'exister à une époque qui survit à la beauté $»^{93}$ - Mallarmé rêve néanmoins d'une grande illumination sous «le ministère du poëte ». Or ce n'est pas un hasard s'il imagine pour cette sortie de l'histoire le cadre d'un « édifice de haut verre » tel le Crystal Palace, autre vision de l'Art achevé depuis l'Exposition universelle de Londres en 1851. À l'époque, le fantasme de doter d'une aura une architecture de verre emblématique du progrès technique a en effet joué un rôle de premier plan, dans la quête antidémocratique d'un nouveau centre symbolique de la cité ; on le retrouve exemplairement dans l'expressionnisme architectural allemand, lorsque Bruno Taut conçoit - dans des termes qui font écho très nettement à ceux de Mallarmé - une « couronne de la ville » [Stadtkrone $]$ en $1919^{94}$.

Mais, que le matériau idéal soit le verre ou la pierre, il importe de soumettre le rêve de cathédrale dont il participe à une «critique de la culture ». Il est difficile en effet de ne pas y déceler, dans le sens de Broch, un «esprit péremptoire [qui] intensifie l'indifférence sociale jusqu'à la cruauté authentique », quand on observe que, suivant le principe du centralisme totalitaire, les nazis ont récupéré le concept de « couronne de la ville ${ }^{95}$. C'est alors un édifice en pierre qu'ils ont voué au ministère de leur Führer «architecte du III ${ }^{\mathrm{e}}$ Reich $»^{96}$. Cependant, ils ont investi la pierre de vertus spirituelles analogues à celles du verre selon Mallarmé et Taut ; «la pierre extraite du sol allemand », dans laquelle le «sang» qui «coul[e]» est «[1]e même » que celui qui coule «dans les veines des hommes », était le matériau idéal de l'architecture nazie, fait pour «garder l'éternité du Reich », car, consubstantielle à la «communauté du peuple allemand ${ }^{97}$, elle donnait pour assise à celle-ci la loi immuable de la nature, douant ainsi d'authenticité son séjour. Or, dès 1934, cette

\footnotetext{
${ }^{93}$ S. Mallarmé, Poèmes en prose : «Le phénomène futur » [1865 (envoi à Henri Cazalis)], dans ibid., t. I, p. 414

${ }^{94}$ Bruno Taut, Une Couronne pour la ville, avec les contributions de Paul Scheerbart, Erich Baron, Adolf Behne, trad. de l'allemand par R. et G. Ballangé et D. Wieczorek, Paris, Éd. du Linteau, 2004.

${ }^{95}$ Je reprends à mon compte l'idée d'Hellmut Lehmann-Haupt que « [l]es nazis se sont emparés, en le corrompant, du concept de couronne de la ville [die Stadtkrone] forgé par Bruno Taut » (Art under a Dictatorship [L'Art sous une dictature], New York, Oxford University Press, 1954, p. 108 ; je traduis). ${ }^{96}$ « On demanda un jour à Hitler pourquoi il n'était pas devenu architecte. Il répondit : "Parce qu'à la place, j'ai décidé de devenir l'architecte du III ${ }^{\mathrm{e}}$ Reich!" ", rapporte Igor Golomštock (L'Art totalitaire: Union soviétique, III Reich, Italie fasciste, Chine, trad. de l'anglais par M. Levy-Bram, Paris, Éd. Carré, 1991, p. 283).

${ }^{97}$ Voir Éric Michaud, Un Art de l'éternité : L'image et le temps du national-socialisme, Paris, Gallimard, coll. «Le temps des images », 1996, p. 113-115.
} 
architecture de pierre faite pour incorporer les individus dans un peuple-un a eu pour pendant les baraquements industriels sordides des camps réservés à l'«antipeuple $» \ldots$

Frédérik Detue 